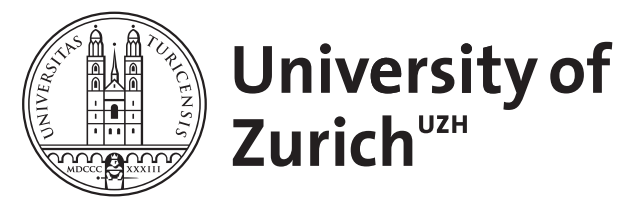

\title{
Ulcus hypertonicum Martorell
}

Hafner, J ; Nobbe, S ; Läuchli, S ; Reutter, D ; Jacomella, V ; Amann-Vesti, B R ; Calcagni, M ; Giovanoli, P ; Böni, Thomas ; Berli, Martin ; Schmid, C ; Speich, R ; Freuch, L E ; Mayer, Dieter

DOI: https://doi.org/10.4414/smf.2014.01857

Posted at the Zurich Open Repository and Archive, University of Zurich ZORA URL: https://doi.org/10.5167/uzh-106018

Journal Article

Published Version

Originally published at:

Hafner, J; Nobbe, S; Läuchli, S; Reutter, D; Jacomella, V; Amann-Vesti, B R; Calcagni, M; Giovanoli, P; Böni, Thomas; Berli, Martin; Schmid, C; Speich, R; Freuch, L E; Mayer, Dieter (2014). Ulcus hypertonicum Martorell. Swiss Medical Forum, (12):242-245.

DOI: https://doi.org/10.4414/smf.2014.01857 


\section{Ulcus hypertonicum Martorell}

Jürg Hafner, Stephan Nobbe, Severin Läuchli, Daniela Reutter, Vincenzo Jacomella, Beatrice Amann-Vesti, Maurizio Calcagni, Pietro Giovanoli, Thomas Böni, Martin Berli, Christoph Schmid, Rudolf Speich, Lars E. Freuch, Dieter Mayer

Interdisziplinäre Wundsprechstunde USZ (Leitung PD Dr. Dieter Mayer), Dermatologische Klinik UniversitätsSpital Zürich

\section{Quintessenz}

- Das Ulcus hypertonicum Martorell gehört zu den häufigen Ursachen chronischer Wunden am Unterschenkel. Die korrekte Diagnose wird aber oft verpasst und fälschlich ein Pyoderma gangraenosum oder eine Vaskulitis diagnostiziert.

Klinisch ist die äusserst schmerzhafte, figurierte Hautnekrose am laterodorsalen Unterschenkel oder über der Achillessehne wegweisend. Eine Ausbreitung der Nekrose im entzündlich-lividen Wundrand ist typisch.

- Histologisch ist die ausgeprägte Arteriolosklerose der Subkutis wegweisend. In mehr als der Hälfte der Fälle wird die Arteriolosklerose von einer Mediakalzinose begleitet.

- Alle Patienten haben eine arterielle Hypertonie, mehr als die Hälfte hat Diabetes Typ 2.

- Die Hälfte der Patienten leidet an einer ipsilateralen PAVK. Der Rest hat eine ausschliessliche Arteriolosklerose der Subkutis.

Die Therapie ist primär chirurgisch: Nekrose abtragen, lokale Unterdrucktherapie, Spalthautverpflanzung. Phasenweise benötigen die meisten Patienten Antibiotika. Oft ist der Krankheitsprozess nach einer erfolgreichen Spalthauttransplantation im Wundrand immer noch progredient. Manche Patienten benötigen daher bis zur Heilung mehrere Operationen.

- Eine Infusionstherapie mit Natrium-Thiosulfat kann die Blutzirkulation und Gewebeoxygenierung verbessern. Natrium-Thiosulfat-Infusionen werden auch in der ähnlich ablaufenden «Calciphylaxis» eingesetzt.

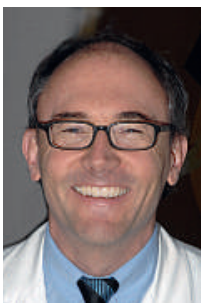

Jürg Hafner

Die Autoren haben keine finanzielle Unterstützung und keine Interessenkonflikte im Zusammenhang mit diesem Beitrag deklariert.
Das Ulcus hypertonicum Martorell wurde 1945 vom spanischen Angiologen Fernando Martorell beschrieben. Praktisch zur gleichen Zeit machten Hines und Farber 1946 an der Mayo Clinic dieselbe Entdeckung und stellten erstmals die ischämisierende Arteriolosklerose der Subkutis als Pathophysiologie zur Diskussion. Sie sprachen vom «Hypertensive Ischemic Leg Ulcer» (HYTILU). Das Ulcus hypertonicum Martorell besteht aus einer rasch progredienten und extrem schmerzhaften Hautnekrose am laterodorsalen Unterschenkel oder über der Achillessehne. 10-15\% aller Patienten mit Ulcus cruris in spezialisierten Wundsprechstunden leiden an dieser Erkrankung. Dennoch ist die Diagnose vielerorts weitgehend unbekannt, und Verwechslungen mit einem Pyoderma gangraenosum oder einer nekrotisierenden Vaskulitis sind sehr häufig. Vor allem die Verwechslung mit einem Pyoderma gangraenosum bedeutet, dass die Patienten von der zielführenden chirurgischen Behandlung ferngehalten werden und dass sie stattdessen eine hochdosierte Immunsuppression erhalten. Unter der Immunsuppression steigt die Gefahr einer Sepsis durch Wundkeime, die bei Patienten mit einem Ulcus hypertonicum Martorell fatal verlaufen kann (bis 10\% Mortalität).

\section{Ätiologie}

Zur Ätiologie des Ulcus hypertonicum Martorell gibt es drei Erklärungsansätze: die ischämisierende Arteriolosklerose, die Mediakalzinose der pathologischen Arteriolen und die Entzündungsvorgänge. Im erkrankten Hautareal haben die Arteriolen eine verbreiterte Gefässwand auf Kosten eines stark reduzierten Lumens. Diese stenosierende Arteriolosklerose ist wahrscheinlich die direkte Ursache der Hautnekrose (Abb. 1 und 2 하). Das Ulcus hypertonicum Martorell kann auch als «Hautinfarkt» aufgefasst werden.

In ungefähr zwei von drei histologischen Schnitten weisen die erkrankten Arteriolen zudem eine Mediakalzinose auf, wie sie von der Mönckeberg-Mediakalzinose der Unterschenkelarterien von Diabetikern oder von terminal niereninsuffizienten Patienten gut bekannt ist. Tatsächlich haben auch rund $60 \%$ der Patienten mit einem Ulcus hypertonicum Martorell nicht ausschliesslich eine arterielle Hypertonie, sondern auch gleichzeitig einen Diabetes Typ 2. Interessant sind die auffälligen Ähnlichkeiten der Histologie des Ulcus hypertonicum Martorell mit der sogenannten «Calciphylaxis» bei terminal niereninsuffizienten oder nierentransplantierten Patienten. Der häufige sekundäre oder tertiäre Hyperparathyroidismus spielt bei der Entstehung der Gefässwandverkalkungen niereninsuffizienter Patienten vermutlich eine wichtige Rolle. In der neusten Diskussion wird dem Serumprotein Alpha2-Heremans-SchmidGlykoprotein (AHSG, Synonym: Fetuin A) bei pathologischen Verkalkungsvorgängen eine Schutzrolle zugeschrieben, weil das AHSG mit Kalzium und Phosphat lösliche Komplexe bilden kann. Die AHSG-Synthese ist von Vitamin Kabhängig, und deshalb empfehlen viele nephrologische Zentren, Patienten mit Calciphylaxis nicht mehr mit Vitamin-K-Antagonisten zu antikoagulieren. Diese Empfehlung wird zunehmend auch auf Patienten mit einem Ulcus hypertonicum Martorell übertragen.

Die Entzündungsvorgänge in der Hautumgebung eines Ulcus hypertonicum Martorell wurden bis jetzt noch wenig untersucht. Eine Arbeit konnte nachweisen, dass in der angrenzenden Haut mehrere proinflammatorische Zytokine hochreguliert sind. Interessant wäre vor allem 


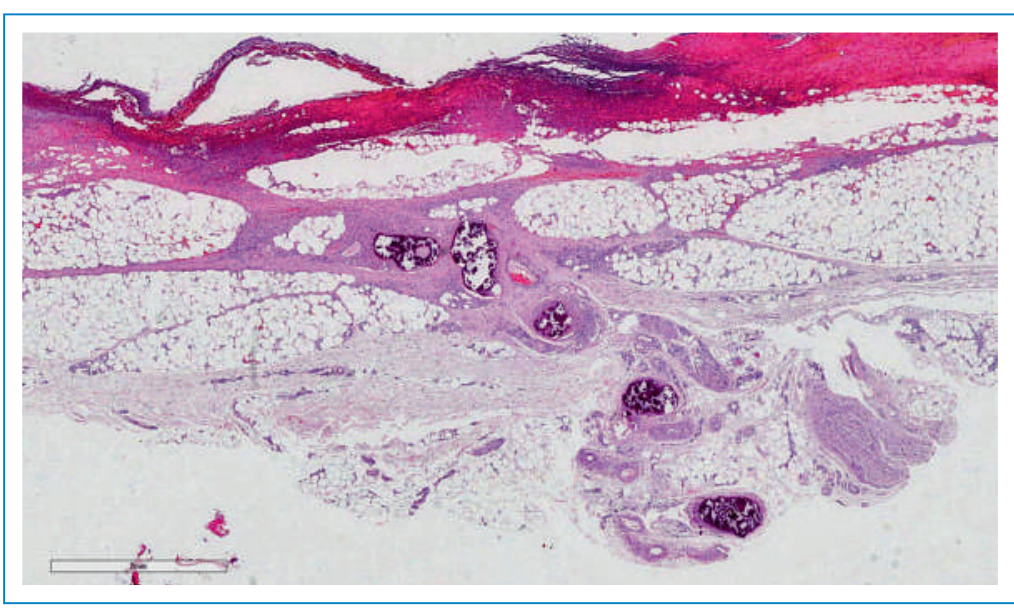

Abbildung 1

Ischämisierende subkutane Arteriolosklerose, Übersicht: Biopsie aus einem Ulcus hypertonicum Martorell. Vollständige Nekrose der Epidermis und Dermis. In der darunter liegenden Subkutis eine Serie pathologischer Arteriolen, mit Mediakalzinose und komplettem Gefässverschluss, an einer Stelle ein organisierter Thrombus im kleinen Restlumen.

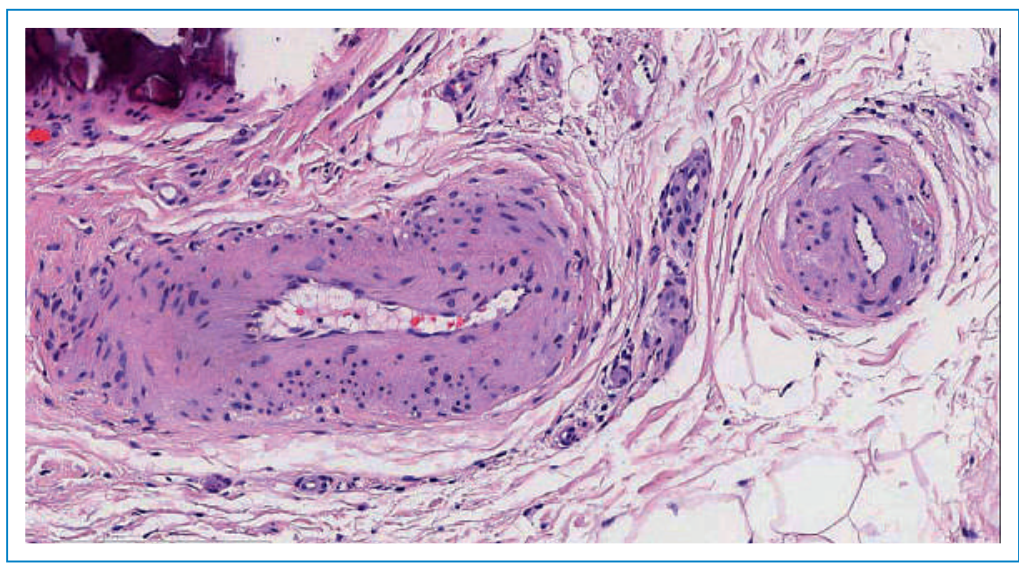

\section{Abbildung 2}

Ischämisierende subkutane Arteriolosklerose, Detail-Aufnahme: subkutane Arteriolen in direkter Umgebung eines Ulcus hypertonicum Martorell. Verdickte Arteriolenwand auf Kosten eines engen Arteriolenlumens, angrenzend angeschnitten eine weitere subkutane Arteriole mit Mediakalzinose (beide histologischen Abbildungen mit freundlicher Genehmigung von der Dermatopathologie, Dermatologische Klinik USZ, Dr. K. Kerl, PD Dr. J. Kamarachev).

Tabelle 1

Vier verwandte Krankheitskomplexe, die sich histologisch durch eine stenosierende subkutane oder akrale Arteriolosklerose auszeichnen.

\begin{tabular}{|c|c|c|}
\hline & Distal & Proximal \\
\hline $\begin{array}{l}\text { Nierenfunktion } \\
\text { normal }\end{array}$ & $\begin{array}{l}\text { Ulcus hypertonicum Martorell } \\
\text { Risikofaktoren: } \\
\text { - Arterielle Hypertonie (100\%) } \\
\text { - Diabetes mellitus Typ } 2(\sim 60 \%) \\
\text { - Orale Antikoagulation }\end{array}$ & $\begin{array}{l}\text { Calciphylaxis bei normaler } \\
\text { Nieren- und «Parathormonfunk- } \\
\text { tion» oder «Eutrophication» } \\
\text { Risikofaktoren: } \\
\text { - Arterielle Hypertonie (100\%) } \\
\text { - Diabetes mellitus Typ } 2(100 \%) \\
\text { - Orale Antikoagulation }\end{array}$ \\
\hline $\begin{array}{l}\text { Terminale } \\
\text { Niereninsuffizienz } \\
\text { oder nierentrans- } \\
\text { plantiert }\end{array}$ & $\begin{array}{l}\text { Calciphylaxis, distale Nekro- } \\
\text { sen } \\
\text { Risikofaktoren: } \\
\text { - Niereninsuffizienz oder Status } \\
\text { nach Nierentransplantation } \\
\text { - Arterielle Hypertonie (häufig) } \\
\text { - Diabetes mellitus Typ } 2 \\
\text { - } 2^{\circ} \text { oder } 3^{\circ} \text { Hyperparathyroi- } \\
\text { dismus } \\
\text { - Orale Antikoagulation }\end{array}$ & $\begin{array}{l}\text { Calciphylaxis, proximale } \\
\text { Nekrosen } \\
\text { Risikofaktoren: } \\
\text { - Niereninsuffizienz oder Status } \\
\text { nach Nierentransplantation } \\
\text { - Arterielle Hypertonie (häufig) } \\
\text { - Diabetes mellitus Typ } 2 \\
\text { - } 2^{\circ} \text { oder } 3^{\circ} \text { Hyperparathyroi- } \\
\text { dismus } \\
\text { - Orale Antikoagulation }\end{array}$ \\
\hline
\end{tabular}

ein vertieftes Verständnis, ob die Entzündung eher eine primäre Ursache des Krankheitsprozesses ist oder ob es sich um eine sekundäre Begleitentzündung handelt. Stünde die Hautentzündung als primäre Ursache am Beginn eines Ulcus hypertonicum Martorell, dann gäbe es eine Grundlage für den Einsatz antientzündlicher Therapien, zum Beispiel mit Biologika.

In Tabelle $1 \Theta$ sind vier Krankheiten zusammengestellt, die wegen ihrer auffälligen Analogie in Klinik und Histologie, wahrscheinlich auch in ihrer Pathogenese, nahe verwandt sind. Sie unterscheiden sich vor allem über die unterschiedlichen Risikofaktoren und Auslöser. Allen gemeinsam ist die arterielle Hypertonie.

Beim Martorell-Krankheitsprozess existiert bei Patienten mit einem metabolischen Syndrom (arterielle Hypertonie, Diabetes Typ 2) im Rahmen einer morbiden Obesitas auch eine proximale Form mit Hautnekrosen an der Innenseite der Oberschenkel, an der Bauchdecken-Fettschürze und an weiteren ausladenden Körperpartien. Man spricht auch von einer «Calciphylaxis bei normaler Nierenfunktion» oder von einer «Eutrophication». Diese Patienten haben eine normale Nierenfunktion. Das nahe verwandte Gegenstück zum Ulcus hypertonicum Martorell und zur Eutrophication ist die Calciphylaxis bei Patienten mit terminaler Niereninsuffizienz und/oder Status nach erfolgreicher Nierentransplantation. Von der Calciphylaxis gibt es eine proximale und eine distale Form.

\section{Klinik}

Die meisten Patienten geben an, das Ulcus hypertonicum Martorell hätte sich innert weniger Tage bis Wochen aus einem lividen Fleck heraus entwickelt. Typisch, jedoch nicht obligat, findet sich in der Hautumgebung eine rankenartige figurierte livide Hautzeichnung, eine Livedo racemosa. Die Ulkus-Umgebung ist meistens ebenfalls schwarz-nekrotisch mit einem angrenzenden düsterroten oder auch hellroten Entzündungssaum.

Das Ulcus hypertonicum Martorell hat eine hoch-charakteristische Prädilektionsstelle: Die laterodorsale Unterschenkel-Region, einschliesslich der Achillessehne (Abb. 3 ㅇ). Manchmal tritt das Ulcus hypertonicum Martorell auch isoliert über der Achillessehne auf. Bloss bei ungefähr 10\% der Patienten sind auch andere Lokalisationen betroffen, zum Beispiel der mediale Unterschenkel. Der Nekrose-Prozess ist typischerweise zentrifugal progredient. Manipulationen, wie zum Beispiel das Setzen einer Lokalanästhesie vor einem scharfen Débridement, können im Rand einen Krankheitsschub auslösen. Wenn ein Ulcus hypertonicum Martorell den Therapieempfehlungen folgend exzidiert, die Wundfläche mit Unterdruck konditioniert und danach Spalthaut transplantiert wird, heilt das Spalthauttransplantat typischerweise im Zentrum auf der Unterschenkelfaszie direkt an, aber die Ulkusumgebung kann dennoch weiterhin in den progredienten Nekroseprozess einbezogen bleiben. Dennoch bedeutet die Spalthautverpflanzung für die Patienten fast immer einen grossen objektiven und subjektiven Fortschritt, denn die Wundfläche wird schlagartig verkleinert, und die Entzündung im Wundrand klingt ab. Nach der Spalthautverpflanzung werden die 
dramatischen Schmerzen meistens innert weniger Tage erträglich. Die Patienten schlafen wieder besser, und die Lebensqualität und Motivation kehren zurück.

\section{Diagnostik}

Das Ulcus hypertonicum Martorell hat eine typische Klinik. Wenn man sich das Bild einmal verinnerlicht hat, wird man die Diagnose kaum noch verpassen. Es handelt sich um eine sehr schmerzhafte und progrediente Hautnekrose am laterodorsalen Unterschenkel oder über der Achillessehne. Vereinzelt können Patienten in der vierten oder fünften Lebensdekade betroffen sein, aber typischerweise sind die Patienten zwischen 60 und 85 Jahre alt, haben eine langjährig gut eingestellte arterielle Hypertonie und in $60 \%$ der Fälle einen Diabetes mellitus Typ II.

Über die Notwendigkeit einer bioptischen Absicherung der Diagnose gehen die Meinungen auseinander. Wir stel-

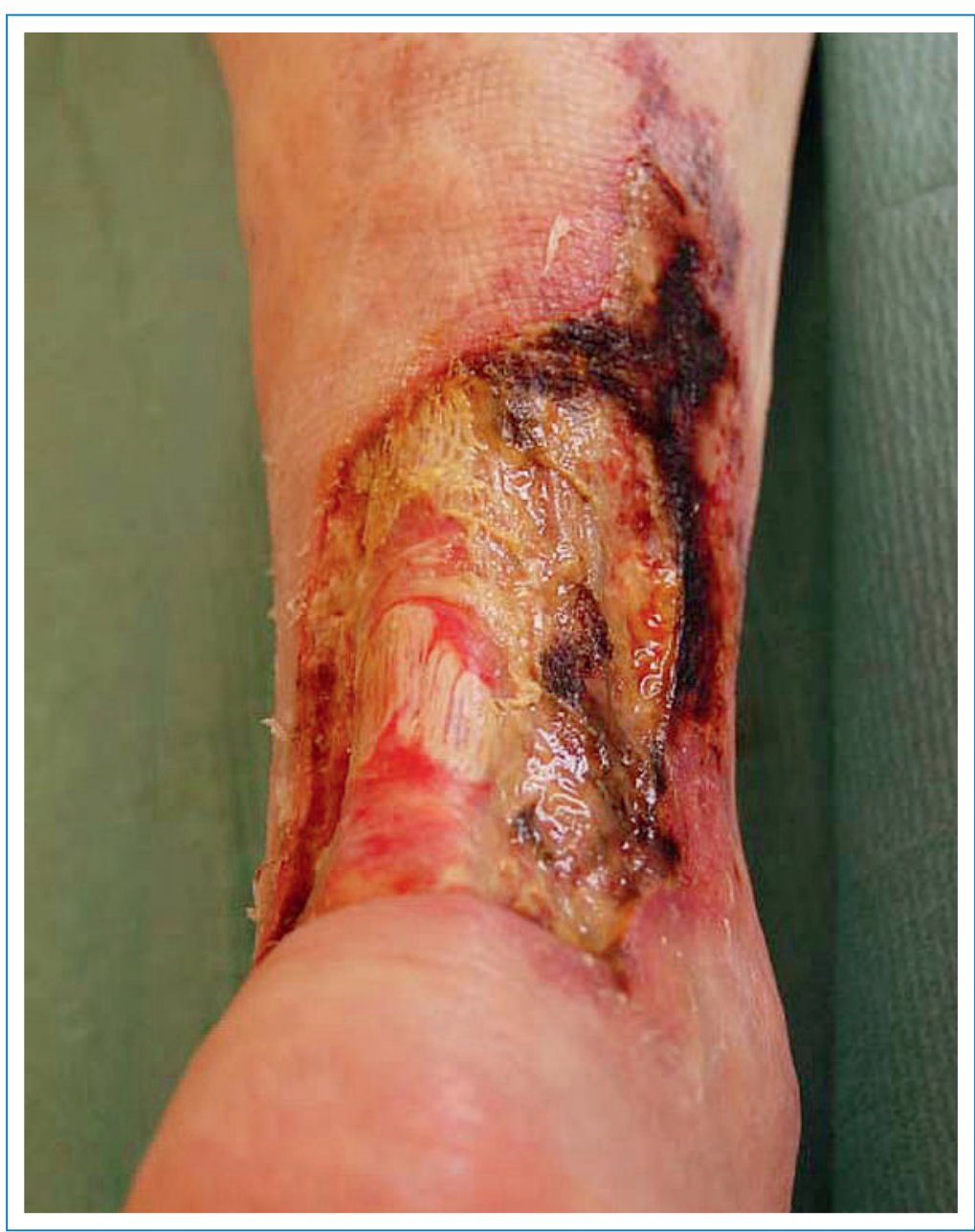

Abbildung 3

80-jähriger Patient mit arterieller Hypertonie, Diabetes mellitus Typ II und normaler Nierenfunktion, der kurz hintereinander zuerst rechts und später links ein Ulcus hypertonicum Martorell entwickelte. Eine periphere arterielle Verschlusskrankheit konnte an beiden Beinen ausgeschlossen werden. Das Bild ziegt die sehr schmerzhafte Hautnekrose am rechten laterodorsalen Unterschenkel. Die Achillessehne liegt frei. Der Wundrand zeigt am laterodorsalen Unterschenkel eine Livedo racemosa. len uns auf den Standpunkt, dass aufgrund der Schwere des Krankheitsbildes und der invasiven therapeutischen Konsequenzen eine Hautbiopsie zur Diagnosesicherung erforderlich ist. Biopsietechnisch bewährt hat sich eine relativ lange, jedoch schmale Probebiopsie eines spindelförmigen Gewebestreifens in Längsachse der Extremität. Die Biopsie beinhaltet zwei bis drei Zentimeter Nekrose und zwei bis drei Zentimeter gesunde Haut, wird also total 4-6 cm lang, jedoch bloss $5 \mathrm{~mm}$ breit, und wird bis auf die Unterschenkel-Faszie entnommen. Dieser schmale Hautstreifen wird intakt belassen und für die Histologie der Länge nach eingekapselt, damit er für die histologische Verarbeitung in seiner gesamten Länge von der Seite her aufgeschnitten werden kann. Damit wird die Möglichkeit eines «sampling errors» praktisch ausgeschaltet. Die Chancen, den pathologischen Prozess - die obliterierende subkutane Arteriolosklerose - vorzufinden, sind unter diesen Voraussetzungen sehr hoch.

Oberflächliche Wundbiopsien wie Stanzbiopsien tragen das Risiko in sich, dass die häufig klinisch gestellte Fehldiagnose Pyoderma gangraenosum auch von histopathologischer Seite vorgeschlagen und somit «bestätigt» wird. In einer oberflächlichen Wundbiopsie sieht man bloss die Nekrosen der oberen Hautschichten und dichte Entzündungsinfiltrate neutrophiler Granulozyten. Es besteht somit die reelle Gefahr einer doppelten klinischpathologischen Fehldiagnose. Es gibt unterschiedliche charakteristische klinische Muster bei den Grund- und Begleiterkrankungen von Patienten mit einem Ulcus hypertonicum Martorell und Patienten mit einem Pyoderma gangraenosum:

- Patienten mit einem Ulcus hypertonicum Martorell sind typischerweise zwischen 60 und 85 Jahren alt und haben eine langjährige, jedoch gut eingestellte arterielle Hypertonie. Rund zwei von drei Patienten haben im Rahmen eines metabolischen Syndroms eine arterielle Hypertonie und einen Diabetes mellitus Typ II, während rund ein Drittel der Patienten schlank ist und ausschliesslich eine arterielle Hypertonie als Grunderkrankung aufweist.

- Patienten mit einem Pyoderma gangraensoum (PG) können jedes Alter haben, sind aber bei Krankheitsausbruch in der Regel jünger als 60-85 Jahre. Die Patienten leiden oft unter weiteren «neutrophilen Krankheiten» wie typischerweise einer entzündlichen Darmkrankheit, aber auch Hidradenitis suppurativa oder an einer hämatoproliferativen Krankheit. Zigarettenrauchen ist mit PG assoziiert. Das ist nicht weiter erstaunlich, denn Rauchen ist ein Auslöser «neutrophiler Krankheiten».

Eine qualitativ einwandfreie angiologische Abklärung der unteren Extremitäten ist unabdingbar. Rund die Hälfte aller Patienten mit einem Ulcus hypertonicum Martorell hat gleichzeitig eine periphere arterielle Verschlusskrankheit im erkrankten Bein, oft begleitet von einer Mediakalzinose der Unterschenkelarterien. Ein Teil der Patienten hat makrovaskuläre Stenosen oder Verschlüsse, die einer interventionellen Therapie (PTA oder gefässchirurgische Revaskularisation) zugänglich sind. 


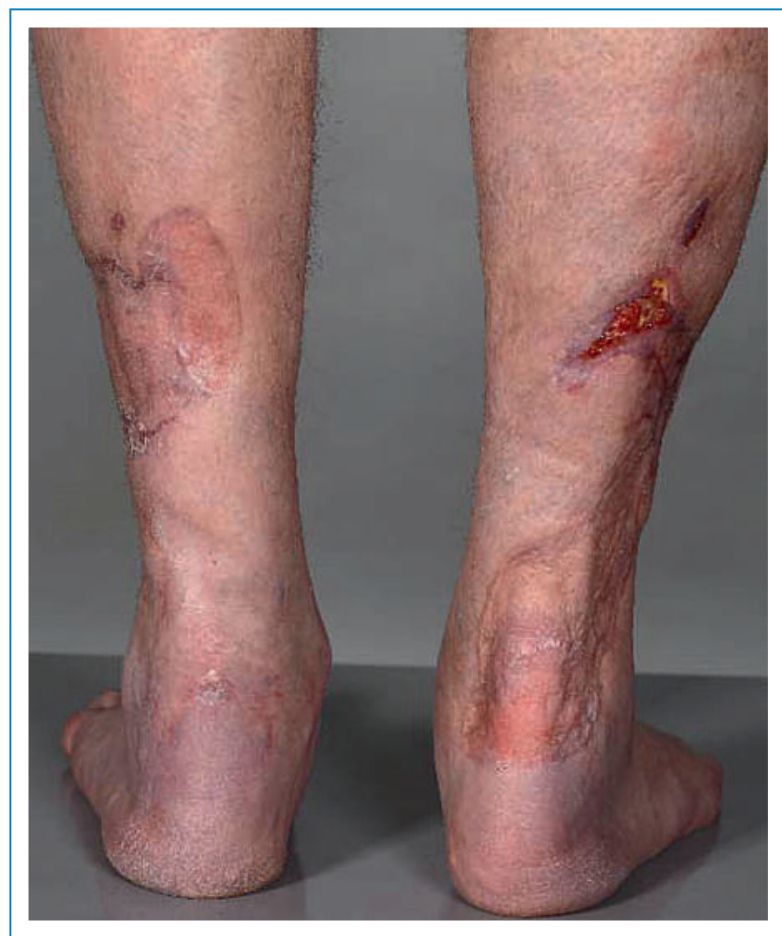

Abbildung 4

Der gleiche Patient wie in Abbildung 1, nach zwölfmonatigem Verlauf. In dieser Zeit mehrere Débridements, mehrere Phasen mit lokaler Unterdrucktherapie, mehrere Phasen mit Antibiotikatherapie, mehrere Spalthauttransplantationen.

\section{Therapie}

Die chirurgische Behandlung führt beim Ulcus hypertonicum Martorell in aller Regel am raschesten zu einer Besserung der fast unerträglichen Schmerzen. Die Hautnekrosen werden bis auf die Unterschenkelfaszie exzidiert, und die Exzisionswunde wird nach Möglichkeit während 6-10 Tagen mit einer lokalen Unterdrucktherapie («Vakuumtherapie») konditioniert. In dieser Phase liegt bei der Mehrzahl der Patienten eine dichte bakterielle Kolonisierung des Ulcus hypertonicum Martorell vor, und bei einem Teil der Patienten finden sich alle Zeichen einer Wundinfektion. Die Beurteilung der Infektsituation erfolgt rein klinisch, denn der CRP-Spiegel kann auch alleine durch die Entzündungsvorgänge rund um den grossflächigen Hautuntergang stark ansteigen, so dass die Interpretation des CRP-Anstiegs schwierig sein kann. Im Zweifelsfall muss in der Phase der Nekrosektomie und Wundkonditionierung eine resistenzgerechte Antibiotikatherapie bis zur Ausbildung von Granulationen durchgeführt werden. Wenn die Wunde aufgrund der Klinik als nicht infiziert beurteilt wird, kann auch bloss ein einmaliger perioperativer Antibiotikaschutz während des scharfen Débridements bzw. der Nekrosen-Exzision durchgeführt werden.

Die Spalthauttransplantate heilen typischerweise im Zentrum auf der Unterschenkelfaszie direkt an, während die Nekrose der Wundumgebung oft noch weiter fortschreitet. Dennoch gehen die Wundschmerzen nach der ersten Spalthautverpflanzung rasch zurück. Beim Ulcus hypertonicum Martorell ist eine Spalthautverpflanzung die wirksamste Analgesie. Sie ist wesentlich wirksamer als alle medikamentösen Schmerztherapien. Wenn möglich soll versucht werden, die neu entstehenden Wundrandnekrosen ambulant und konservativ durch umschriebene Débridements und eine geeignete Lokaltherapie mit synthetischen Verbänden ganz zur Abheilung zu bringen. Ein Teil der Patienten benötigt jedoch mehrere chirurgische Folgeeingriffe, bis die Krankheit zum Stillstand kommt und sämtliche Wunden abheilen (Abb. 4 하).

Wenn der Prozess der ischämischen Hautnekrosen sehr weit um sich greift und grosse Flächen betroffen sind, muss der Einsatz von Natrium-Thiosulfat-Infusionen (10 Gramm Na-Thiosulfat in 100 ml Trägerlösung, Infusionsdauer 2 Stunden) auf täglicher Basis oder dreimal pro Woche diskutiert werden. Natrium-Thiosulfat löst Kalk auf. Man geht davon aus, dass die Infusionen die Blutzirkulation der versteiften und grossenteils verkalkten Arteriolen verbessern. Sobald der klinische Effekt eintritt, und dies kann schon nach wenigen Tagen der Fall sein, aber auch mehrere Wochen dauern, wird die Haut klinisch weniger livid und mit der Zeit wieder «rosig», und in den zuvor avitalen Wundflächen entsteht zusehends Granulationsgewebe. Unter der Natrium-Thiosulfat-Therapie entsteht eine Azidose, die Nausea provozieren kann. Die Nausea kann beispielsweise mit einer vorangehenden Verabreichung von Ondansetron wirksam bekämpft werden. Aufgrund der bis heute gemachten Erfahrungen scheint die Natrium-ThiosulfatTherapie auf die Knochendichte keinen Einfluss zu haben. Möglicherweise können Natrium-Thiosulfat-Infusionen lebensrettend sein, vor allem bei Patienten mit sehr ausgedehnten Hautnekrosen infolge einer hypertensiven Arteriolosklerose oder einer Calciphylaxis.

Die Behandlung von Patienten mit einem Ulcus hypertonicum Martorell ist für alle Beteiligten - Patienten wie Betreuende - eine grosse Herausforderung. In den letzten Jahren ist es glücklicherweise gelungen, aufgrund der Fortschritte im Verständnis und der daraus abgeleiteten Therapiemassnahmen den schwerkranken Betroffenen wesentlich rascher zu helfen.

\section{Korrespondenz:}

Prof. Dr. med. Jürg Hafner

Dermatologische Klinik

UniversitätsSpital

CH-8091 Zürich

juerg.hafner[at]usz.ch

\section{Weiterführende Literatur}

- Hafner J, Nobbe S, Partsch H, et al.: Martorell hypertensive ischemic leg ulcer. A model of ischemic subcutaneous arteriolosclerosis. Arch Dermatol. 2010;146:961-8.

- Vuerstaek JD, Reeder SW, Henquet CJ, Neumann HAM: Arteriolosclerotic ulcer of Martorell. J Eur Acad Dermatol Venereol. 2010;24:867-74

- Alavi A, Mayer D, Hafner J, Sibbald RG. Martorell hypertensive ischemic leg ulcer: an underdiagnosed entity. Advances Skin Wound Care. 2012;25:563-72.

- Kalajian AH, Malhotra PS, Callen JP et al.: Calciphylaxis with normal renal and parathyroid function: not as rare as previously believed. Arch Dermatol. 2009;145:451-8.

- Hackett BC McAleer MA, Sheehan G, et al.: Calciphylaxis in a patient with normal renal function: response to treatment with sodium thiosulfate. Clin Exp Dermatol. 2009;34:39-42.

- Nigwekar SU, Brunelli SM, Meade D, Wang W, Hymes J, Lacson E Jr: Sodium thiosulfate for calcific uremic arteriolopathy. Clin J Am Soc Nephrol. 2013;8:1162-70.

- Giot JP, Paris I, Levillain P, et al.:Involvement of IL-1 and oncostatin M in acanthosis associated with hypertensive ischemic leg ulcer. Am J Pathol. 2013;182:806-18. 